\title{
Effectiveness of Cardiac Rehabilitation on Health-related Quality of Life in Patients with Myocardial Infarction in Pakistan
}

\author{
Zia Ul-Haq1,4, Daud Khan1, Aliya Hisam², Yasar Mehmood Yousafzai1, Shazia Hafeez³, \\ Fatima Zulfiqar33, Adnan Mahmood Gul3, Mohammad Hafizullah¹ and Jill Pell4 \\ ${ }^{1}$ Institute of Public Health \& Social Sciences, Khyber Medical University, Peshawar, Pakistan \\ ${ }^{2}$ Department of Community Medicine, Army Medical College, National University of Medical Sciences, Rawalpindi, Pakistan \\ ${ }^{3}$ Department of Preventive Cardiology, Lady Reading Hospital, Peshawar, Pakistan \\ ${ }^{4}$ Institutes of Health \& Wellbeing, University of Glasgow, UK
}

\begin{abstract}
Objective: To find out the effectiveness of cardiac rehabilitation in patients with myocardial infarction in Pakistan.

Study Design: Randomised controlled trial.

Place and Duration of Study: Cardiac Rehabilitation Unit, Lady Reading Hospital, Peshawar, Pakistan, from July to December 2016.

Methodology: Patients suffering first myocardial infarction (MI) were randomly allocated to usual care or cardiac rehabilitation in a 1:1 ratio. Cardiac rehabilitation comprised two phases: 1-2 weeks during hospital stay followed by 6-7 weeks outpatient structured exercise programme. Two generic health related quality of life (HRQLL) outcomes (General Health Questionnaire (GHQ) and Self-Rated Health (SRH)) and one post-MI specific tool (MacNew QLMI) were measured at baseline and at 8 weeks follow-up among both groups. Lower SRH and GHQ scores and higher MacNew QLMI scores indicate better health status. Data were analysed using STATA 14.

Results: Out of 206 participants, $195(94.6 \%)$ were analysed at the end of trial. The mean age was $53 \pm 8.3$ years. In the cardiac rehabilitation group, the mean $\mathrm{SRH}$ score changed from $3.97 \pm 0.9$ at baseline to $2.36 \pm 0.8$ at follow-up ( $p<0.001$ ). The mean GHQ of the cardiac rehabilitation group was $21.26 \pm 5.5$ at baseline and it decreased significantly to $7.43 \pm 4.2$ at follow-up $(p<0.001)$. The MacNew QLMI of the cardiac rehabilitation group increased from $3.61 \pm 1.07$ to $5.62 \pm 0.5$ $(p<0.001)$. The multivariate regression of all three HRQoL measures confirmed better HRQoL following cardiac rehabilitation compared with usual care (all $p<0.001$ ).

Conclusion: Cardiac rehabilitation following MI was effective in terms of improving HRQL and can be implement in Pakistan as it produced significant improvements in HRQoL.
\end{abstract}

Key Words: Cardiac rehabilitation, Rehabilitation, Myocardial infarction, Heart attack, Quality of life, Health-related quality of life, Self-reported health, General health questionnaire, MacNew quality of life after myocardial infarction.

How to cite this article: Haq ZU, Khan D, Hisam A, Yousafzai YM, Hafeez S, Zulfiqar F, Gul AM, Hafizullah M, Pell J. Effectiveness of cardiac rehabilitation on health-related quality of life in patients with myocardial infarction in Pakistan. J Coll Physicians Surg Pak 2019; 29(9):803-9.

\section{INTRODUCTION}

Globally, the leading cause of deaths is cardiovascular disease (CVD). A total of $19 \%$ deaths are a result of CVDs, with age standardised death rates of 255 and 295 per 100,000 for males and females, respectively. ${ }^{1}$ In developing countries with high population density, the prevalence of risk factors such as physical inactivity, poor diet, obesity, smoking, type 2 diabetes, hypertension, and abnormal lipid profile is increasing. As a result, the prevalence of non-communicable diseases, such as CVD, is increasing in developing countries where nearly one quarter $(24.3 \%)$ of people above the

Correspondence to: Prof. Dr. Zia Ul-Haq, Institute of Public Health \& Social Sciences, Khyber Medical University, Peshawar, Pakistan

E-mail:drzia@kmu.edu.pk

Received: January 14, 2019; Revised: March 04, 2019;

Accepted: May 23, 2019 age of 18 years have been reported as hypertensive. ${ }^{2}$ Similarly, $25 \%$ of people above the age of 40 years were found to be suffering from coronary artery diseases (CAD).3,4

Cardiac rehabilitation has been shown to be clinically effective as secondary prevention following myocardial infarction (MI) in developed countries. It aims to improve the patient's physical, psychological and social wellbeing, and produce behavioral changes that reduce the risk of disease progression and future events. ${ }^{5}$ Cardiac rehabilitation programmes usually involve a number of complementary interventions including exercise, nutrition and diet counselling, psychological support, education on risk factors, and medication compliance. 6 The impact of psychotherapeutic interventions, which include counselling, relaxation training and stress management, are unclear with some studies suggesting they are effective, ${ }^{7}$ and other studies showing weaker or no effects. 8,9 
In Pakistan, the usual care for myocardial infarction does not have standardised cardiac rehabilitation. There is a lack of studies on whether secondary prevention of CVD is effective in developing countries, particularly South Asian.

This study aimed to evaluate the impact of cardiac rehabilitation on the health-related quality of life of patients admitted for myocardial infarction to a tertiary healthcare facility in the Khyber Pakhtunkhwa province of Pakistan.

\section{METHODOLOGY}

This parallel randomised controlled trial was designed using the 2010 CONSORT (Consolidated Standards of Reporting Trials) guidelines. Ethical approval was obtained from the Ethical Board and the trial was registered with Australian New Zealand Clinical Trials Registry (ANZCTR) with the trial registration number ACTRN12617000832370.10 The study was undertaken in Cardiac Rehabilitation Unit, Lady Reading Hospital, Peshawar, Pakistan, from July to December 2016. The primary outcome measure, that is MacNew QLMI, was used for sample size estimation in STATA version 14.

A sample size of 79 per group in ratio of $1: 1$ was calculated by two independent mean tests (control group; 5.2 and intervention group 6.1), 11 assuming a variability of 2 in groups, a power of $80 \%$ and a significance level of $5 \%$. To allow for a predicted $30 \%$ dropout or lost to follow-up rate from adverse events, the sample size has been increased to 103 per group, recruiting a total of 206 participants. A research associate was trained regarding the randomisation, and was handed over the allocation concealment responsibility. The sampling technique used for this trial was permuted-block randomisation. Blocking is a method of restricted randomisation that keeps a balance between the two groups at the end of every block. A sealed envelope was used to allocate each patient into either usual care or cardiac rehabilitation.

The inclusion criteria were patients of 70 years or below with a first $\mathrm{Ml}$ receiving their primary treatment in a cardiology ward. Exclusion criteria were participants with any type of documented mental illness or any type of physical disability and those who were unable to communicate or provide informed consent. Patients were also excluded if they had a resting systolic blood pressure $>200 \mathrm{~mm} \mathrm{Hg}$, diastolic blood pressure $>100$ $\mathrm{mm} \mathrm{Hg}$, ongoing chest pain at rest, decompensated heart failure, severe symptomatic valvular heart disease, complex ventricular arrhythmia, resting paroxysmal supraventricular tachycardia, complex arrhythmia induced by exercise, third degree atrioventricular block without permanent pacemaker, endocarditis, pericarditis or arterial thrombo-embolism, or were unable to perform physical exertion for other reasons.
After patients were diagnosed with MI by a cardiologist, they were recruited from the cardiology ward, baseline information recorded and the patients were then randomised into usual care or usual care plus cardiac rehabilitation. The usual care group received only the standard communication from the cardiologist and routine follow-up care. The routine follow-up care includes brief counselling session regarding their health, medicine prescription and follow-up advise. They did not receive the structured counselling session as the intervention group. They were prescribed cardiac medications according to their condition and needs, judged by their cardiologist. No attempt was made to standardise the care of usual care arm as they were treated according to their needs as assessed by their cardiologists. Their follow-up was planned usually between 2-6 weeks post-discharge by their cardiologists. Cardiac rehabilitation was medically supervised and run by a multidisciplinary team comprising one cardiac consultant, two trained nurses, 1 physiotherapist, and 1 dietitian. Cardiac rehabilitation was delivered in the hospital premises over a period of 8 weeks and comprised the following 2 phases:

Phase 1 was delivered over the first 1-2 weeks while the participant was still an inpatient. Phase 2 commenced immediately following phase 1 and comprised a 6-7 weeks structured exercise programme. The details of cardiac rehabilitation is available at ANZCTR10.

Self-rated health $(\mathrm{SRH})$ was determined via a single question: "In general, would you say that your health is excellent, very good, good, fair, or poor?" with five options from excellent to poor (Score 1-5). A lower score indicated better health status. ${ }^{12}$ Its Urdu version was available and used. The standardised General Health Questionnaire (GHQ) proforma was used. Its score range is $0-36$. A score of $<15$ is interpreted as no distress; whereas, $>15$ shows distress. Scores of $>20$ indicated severe problems along with psychological distress. ${ }^{13}$ Its Urdu version was used. The MacNew Quality of Life after Myocardial Infarction (MacNew QLMI) index consists of 27 questions with each providing 7 response options. Higher scores indicated better health. A global score was calculated as the average over all items. ${ }^{14}$ Its Urdu version was available and used.

Patients with $\mathrm{MI}$, who received initial treatment in the ward, were allocated using the permuted-block randomisation method, by computer generated random numbers (using STATA- stata@stata.com) with different block sizes (4, 6 and 8$)$ using sequentially numbered opaque and sealed envelopes (SNOSE). First baseline assessment was conducted and then random allocation was performed and the patients were randomly allocated to either usual care (controls) or cardiac rehabilitation (intervention group). Only cardiac rehabilitation patients 
were asked to come every week for consecutive 6-7 weeks, while usual care were given the routine care.

Both groups' face-to-face pretested questionnaires were asked through trained staff at baseline and at the end of 8 week follow-up regarding demographic information (age, gender, education, monthly income, marital status), lifestyle risk factor (smoking status), self-reported physician-diagnosed comorbidities (hyper-tension, diabetes, chronic obstructive pulmonary disease, cancers), SRH, GHQ, and MacNew QLMI. Anthropometric measurements included height and weight measured; and body mass index (BMI) was calculated using the standard protocol. ${ }^{15}$ The HRQoL measures were the primary outcome variable. Advanced Studies and Research Board (ASRB) and Ethical Committee approved the trial.

Data were entered and analysed in STATA 14. Frequencies and percentages were calculated for categorical variables such as age, BMI, smoking status, and comorbidities and Chi-square tests used to compare the two treatment arms. Means and standard deviation (SD) were calculated for continuous variables, such as $\mathrm{SRH}, \mathrm{GHQ}$ and MacNew, and independent sample t-tests used to compare the two groups at follow-up. Paired t-tests were used to compare follow-up with baseline within the usual care and cardiac rehabilitation groups. Chi-square test was applied to find out association between the two group (usual care and cardiac rehabilitation) and demographic variables.

Linear multivariate regression analysis was conducted to predict any change in the HRQoL measures among both groups at follow-up period. In model 1, age along with smoking status were adjusted while in model 2 , age, gender, educational and smoking status, body mass index, monthly income and comorbidity were adjusted. The tests of significance were two-tailed. A p-value of $<0.05$ was taken as statistically significant.

\section{RESULTS}

A total of 206 participants were registered in the study with 1:1 ratio in both groups; out of which, 195 (94.6\%) were finally analysed at the end of the trial. Three patients in the cardiac rehabilitation arm, and five in the usual care group, died prior to completion of the study. Similarly, one patient was lost to follow-up in cardiac rehabilitation group and two in usual care group (Figure 1).

The participants' mean age was $53.6 \pm 8.3$ years. In both groups, the majority of participants were in the 45-60 years of age category, male, and had no formal education. Patients in the cardiac rehabilitation arm were significantly more likely to have formal education and were significantly less likely to smoke or have comorbidities (Table I).

Table I: Characteristics of participants by trial arm.

\begin{tabular}{|c|c|c|c|c|}
\hline & $\begin{array}{l}\text { Usual care }(n=96) \\
n(\%)\end{array}$ & $\begin{array}{l}\text { Cardiac rehabilitation }(\mathrm{n}=99) \\
\mathrm{n}(\%)\end{array}$ & $p$-value & $\begin{array}{c}\text { Overall }(n=195) \\
n(\%)\end{array}$ \\
\hline \multicolumn{5}{|l|}{ Age (years) } \\
\hline$<45$ & $10(10.42)$ & $14(14.14)$ & 0.552 & $24(12.31)$ \\
\hline $45 \&<=60$ & $54(56.25)$ & $58(58.59)$ & & $112(57.44)$ \\
\hline$>60$ & $32(33.33)$ & $27(27.27)$ & & $59(30.26)$ \\
\hline \multicolumn{5}{|l|}{ Gender } \\
\hline Male & $69(71.88)$ & $81(81.82)$ & 0.099 & $150(76.92)$ \\
\hline Female & $27(28.13)$ & $18(18.18)$ & & $45(23.08)$ \\
\hline \multicolumn{5}{|l|}{ Education status } \\
\hline No formal education & $75(78.13)$ & $53(53.54)$ & 0.001 & $128(65.64)$ \\
\hline Matriculate & $17(17.71)$ & $32(32.32)$ & & $49(25.13)$ \\
\hline Above Matric & $4(4.17)$ & $14(14.14)$ & & $18(9.23)$ \\
\hline \multicolumn{5}{|l|}{ Smoking status } \\
\hline Non-smoker & $77(80.21)$ & $62(62.63)$ & 0.013 & $139(71.28)$ \\
\hline Ex-smoker & $13(13.54)$ & 19 (19.19) & & $32(16.41)$ \\
\hline Current smoker & $6(6.25)$ & $18(18.18)$ & & $24(12.31)$ \\
\hline \multicolumn{5}{|l|}{ Body mass index } \\
\hline Normal & $42(43.75)$ & $38(38.38)$ & 0.265 & $80(41.03)$ \\
\hline Overweight & $42(43.75)$ & $40(40.40)$ & & $82(42.05)$ \\
\hline Obese & $12(12.5)$ & $21(21.21)$ & & $33(16.92)$ \\
\hline \multicolumn{5}{|l|}{ Monthly income (PKR) } \\
\hline$<8,500$ & $15(15.63)$ & $13(13.13)$ & 0.071 & $28(14.36)$ \\
\hline $8,500-20,000$ & $33(34.38)$ & $50(50.51)$ & & $83(42.56)$ \\
\hline$>20,000$ & $48(50)$ & $36(36.36)$ & & $84(43.08)$ \\
\hline \multicolumn{5}{|l|}{ Comorbidity } \\
\hline No & $23(23.96)$ & $52(52.53)$ & $<0.001$ & $75(38.46)$ \\
\hline Yes & $73(76.04)$ & $47(47.47)$ & & $120(61.54)$ \\
\hline
\end{tabular}

${ }^{*}$ Chi-square test is applied to find out association between the two groups (usual care and cardiac rehabilitation) and demographic variables; $n$, population; PKR, Pakistani rupees. 
Table II: Health-related quality of life measures by trial arm.

\begin{tabular}{|c|c|c|c|c|c|c|}
\hline & \multicolumn{3}{|c|}{ Usual care $(n=96)$} & \multicolumn{3}{|c|}{ Cardiac rehabilitation $(n=99)$} \\
\hline & $\begin{array}{c}\text { Baseline } \\
\text { Mean } \pm S D\end{array}$ & $\begin{array}{l}\text { Follow-up } \\
\text { Mean } \pm S D\end{array}$ & $p$-value* & $\begin{array}{l}\text { Baseline } \\
\text { Mean } \pm S D\end{array}$ & $\begin{array}{l}\text { Follow-up } \\
\text { Mean } \pm S D\end{array}$ & p-value* \\
\hline $\begin{array}{l}\text { SRH } \\
\text { p-value ** }\end{array}$ & $3.9 \pm 0.07$ & $4.06 \pm 0.06$ & 0.042 & $3.97 \pm 0.9$ & $2.3 \pm 0.8$ & $\begin{array}{l}<0.001 \\
<0.001\end{array}$ \\
\hline $\begin{array}{l}\text { GHQ } \\
\text { p-value ** }\end{array}$ & $18.71 \pm 4.3$ & $20.9 \pm 5.2$ & $<0.001$ & $21.2 \pm 5.5$ & $7.4 \pm 4.2$ & $\begin{array}{l}<0.001 \\
<0.001\end{array}$ \\
\hline $\begin{array}{l}\text { MacNew QLMI } \\
\text { p-value }{ }^{\star \star}\end{array}$ & $3.9 \pm 0.5$ & $3.8 \pm 0.5$ & 0.010 & $3.6 \pm 1.07$ & $5.6+0.5$ & $\begin{array}{l}<0.001 \\
<0.001\end{array}$ \\
\hline
\end{tabular}

SRH, range is 1-5: Lower score indicates better health status; GHQ, range is 0-36, Lower score indicates better health status; MacNew QLMI, range of global score is 1-7, Higher score signifies better health status; ${ }^{*}$ Paired $t$-test, applied at baseline and follow-up of each group; ${ }^{* *}$ independent sample t-test is applied between the follow-up of two groups (usual care and cardiac rehabilitation); $n=$ population.

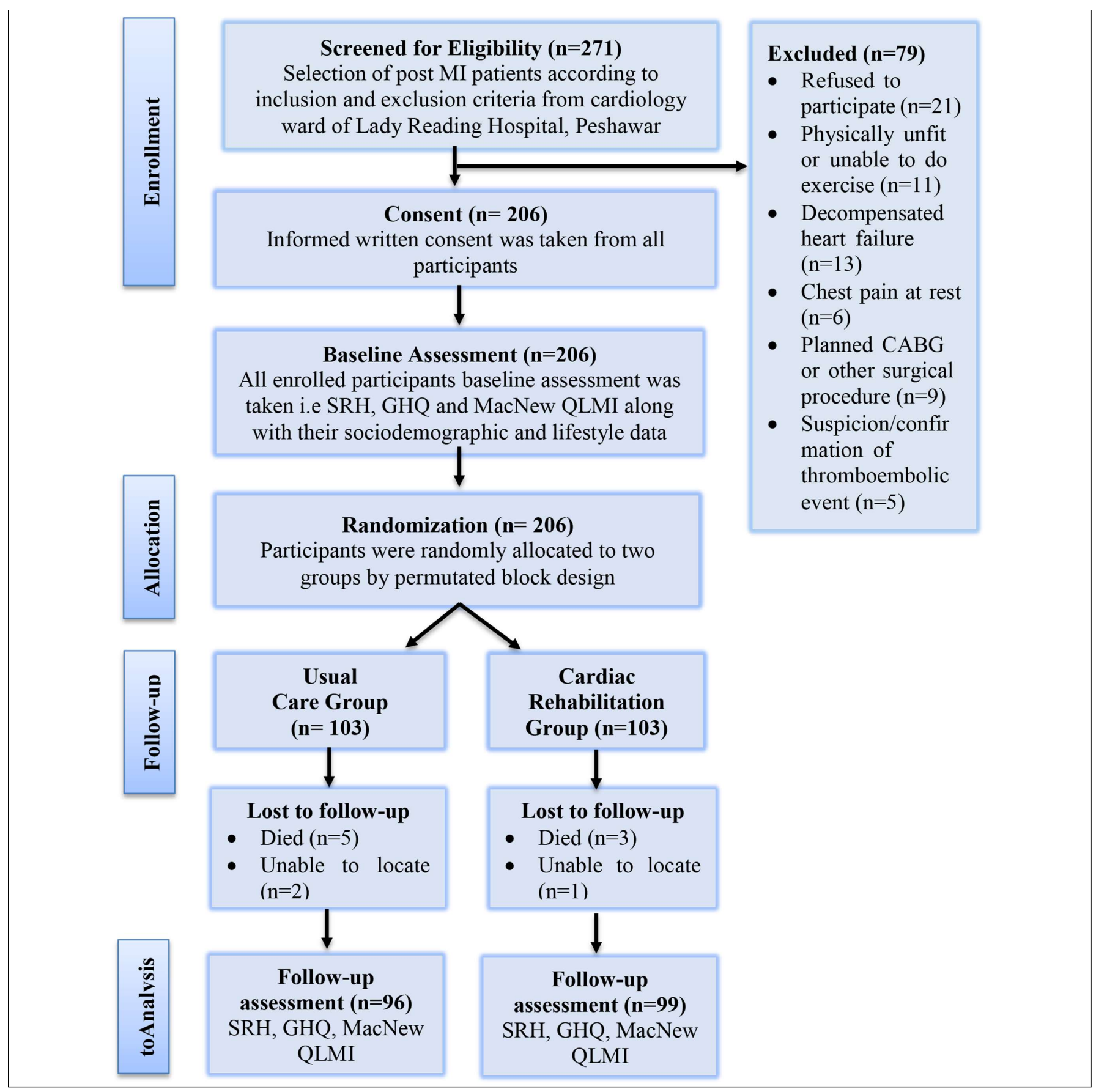

Figure 1: Flow diagram showing participants' enrollment per CONSORT guidelines. 

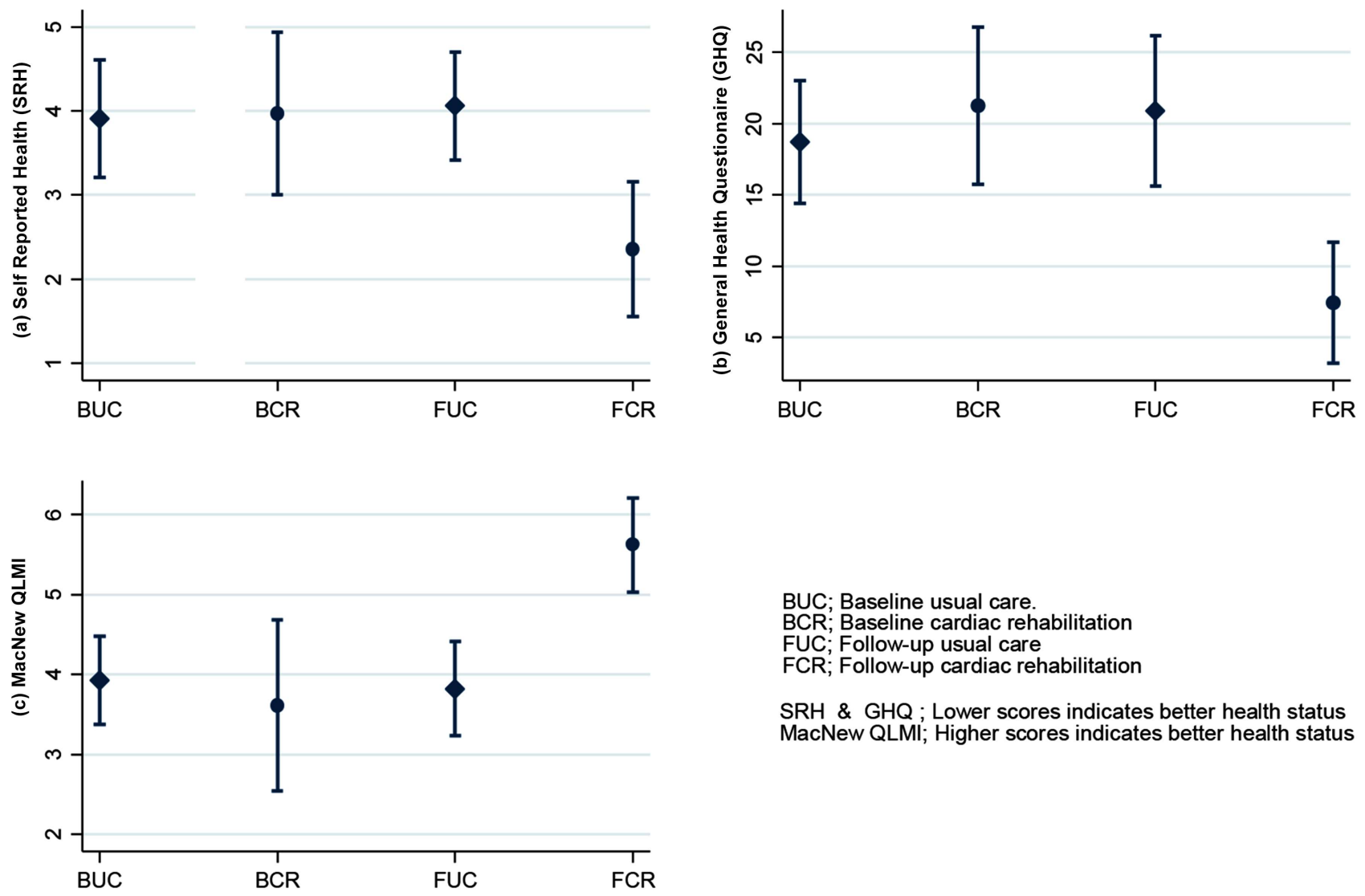

BUC; Baseline usual care.

BCR; Baseline cardiac rehabilitation

FUC; Follow-up usual care

FCR; Follow-up cardiac rehabilitation

SRH \& GHQ ; Lower scores indicates better health status MacNew QLMI; Higher scores indicates better health status

Figure 2: Health-related quality of life among usual and cardiac rehabilitation groups (a) SRH (b) GHQ (c) QLMI.

In the usual care group, the mean SRH scores were $3.91 \pm 0.07$ at baseline and $4.06 \pm 0.06$ at follow-up. This increase was significant $(p=0.04)$ showing deterioration of HRQoL. In the cardiac rehabilitation group, the mean $\mathrm{SRH}$ score changed from $3.97 \pm 0.9$ at baseline to 2.36 \pm 0.8 at follow-up, showing a significant improvement in HRQoL after cardiac rehabilitation $(p<0.001)$. The follow-up SRH scores were significantly different between the two treatment arms $(p<0.001$, Table II).

In the model 1 of multivariate regression analysis, the cardiac rehabilitation group still experienced significantly increased HRQoL at follow-up, after adjusting for age and smoking status $\left(r^{2}=0.58,-1.46, p<0.001\right)$. In model 2 , after further adjusting for gender, educational status, $\mathrm{BMI}$, monthly income and comorbidity, the increase was greater $\left(r^{2}=0.60, p<0.001\right)$.

Among the usual care group, mean GHQ scores at baseline and follow-up were $18.71 \pm 4.3$ and $20.91 \pm 5.2$, respectively. Among the cardiac rehabilitation group, the mean $\mathrm{GHQ}$ was $21.26 \pm 5.5$ at baseline and it decreased significantly to $7.43 \pm 4.2$ at follow-up $(p<0.001)$, showing that $\mathrm{HRQ}$ oL had improved. At follow-up, HRQoL was significantly better in the cardiac rehabilitation group than the usual care group $(p<0.001$, Table II).
In the model 1 multivariate regression analysis, $\mathrm{HRQ}$ oL had increased significantly at follow-up in the cardiac rehabilitation group after adjusting for age and smoking status $\left(r^{2}=0.67, p<0.001\right)$. In model 2, after further adjusting for gender, educational status, body mass index, monthly income and comorbidity, there was still a significant increase in HRQoL $\left(r^{2}=70, p<0.001\right)$. In the usual care, the mean MacNew QLMI score fell slightly from $3.93 \pm 0.5$ at baseline to $3.82 \pm 0.5$ at follow-up $(p<0.01)$. In contrast, in the cardiac rehabilitation group, it increased from $3.61 \pm 1.07$ to $5.62 \pm 0.5(p<0.001)$. When both groups were compared at follow-up, the difference was strongly significant $(p<0.001$, Table II). In the model 1 , the cardiac rehabilitation group at the follow-up period showed increase in the HRQoL, after adjusting for age and smoking status $\left(r^{2}=0.70, p<0.001\right)$ as increase MacNew QLMI indicates better quality of life. In model 2, after adjusting for gender, age, educational and smoking status, body mass index, monthly income and comorbidity, there was still a significant improvement in $\mathrm{HRQoL}\left(r^{2}=0.72, p<0.001\right)$. The detail description of the generic and specific HRQoL measures are shown in Figure 2. 


\section{DISCUSSION}

This randomised controlled trial study demonstrated that a hospital-based cardiac rehabilitation programme could improve the HRQoL among patients admitted with myocardial infarction in Pakistan and that the intervention significantly improved the HRQoL of patients.

The age distribution of the patients was comparable to other studies studying patients with CAD. The proportion of men as compared to the women was larger in our study. The same trend was noted in many other studies carried out for the assessment of HRQoL in patients with CVD. 16,17 The gender difference is notable. In our study, higher use of health services by males might be attributed for increase in cardiac rehabilitation uptake.

In this study, HRQoL was significantly better in patients receiving 4 weeks of comprehensive cardiac rehabilitation in comparison to the patients in control group. A study of 8 weeks cardiac rehabilitation on MI patients showed considerably better enhancement in overall physiological outcomes in the group participating in cardiac rehabilitation programme compared with the control group. An appreciable improvement in short form-36 health status subscales (4 out of 8 i.e. physical functioning, rolephysical, general health, and vitality) was noted in the intervention group (cardiac rehabilitation) compared with the control group (non-cardiac rehabilitation). ${ }^{18}$

Grace et al. have reported that cardiac rehabilitation could result in an enhancement in HRQoL and improve the anxiety level of the patients. They have also found that cardiac rehabilitation has positive effects on the signs of depression. 19 In one meta-analysis, conducted by Samartzis et al., data from 1,074 subjects (intervention group) against 1,106 subjects (control group) were analysed. The authors observed that cardiac rehabilitation enhanced HRQoL of the patients by improving their mental and psychological status $(p<0.05) .20$ The above findings are consistent with the current study.

A clinical trial carried out by Chan et al. in Hong Kong, resulted in enhancement in patients' HRQoL, irrespective of the intervention group though the dissimilarity was insignificant. The outcomes dissimilarity with those of the current study, especially in the control group, may be due to the dissimilarity in the sample size and the duration of study (six months), in addition to high number of dropout rate of the subjects in the study. ${ }^{21}$

A study done in Portugal, by Bettencourt et al., studied 31 subjects in intervention group and 95 subjects in control, have found that cardiac rehabilitation showed no significant outcome on the areas of HRQoL, excluding two aspects (excitement and general health). This could be due to an unequal allotment of subjects in two groups. ${ }^{22}$ A systematic review article, published in 2010 , showed that both home-based and center-based cardiac rehabilitation significantly improved HRQoL. ${ }^{23}$
This study has shown for the first time in Pakistan that cardiac rehabilitation after $\mathrm{MI}$ significantly improves HRQoL. The physiological, psychological and specific HRQoL measures were all improved in post-MI patients after comprehensive cardiac rehabilitation programme. However, our results are not without limitations. This was a single centre study with a relatively short follow-up period and the study should be repeated to determine if the benefits are maintained over longer follow-up.

A limitation of this study is that as it was the first study of its kind in Pakistan so the study results cannot be generalised to Pakistani, population or to a group of patients conducted in any hospital settings. The duration of intervention was only 8 weeks and if it was for a longer period of time, the result would be clearer. As participants of this study were only from a single unit of one hospital, so the sample was not representative to cover all those subjects attending private clinics and other hospitals. One more limitation of the study was that it was not titrated, and adherence was also not measured.

\section{CONCLUSION}

Cardiac rehabilitation following MI was effective in terms of improving HRQoL and can be implemented in Pakistan as it produced significant improvements in HRQoL.

\section{ETHICAL APPROVAL:}

Ethical approval was obtained from the Ethical Board and the trial was registered with Australian New Zealand Clinical Trials Registry (ANZCTR) with the trial registration number ACTRN12617000832370.

\section{CONFLICT OF INTEREST:}

Authors declared no conflict of interest.

\section{AUTHORS' CONTRIBUTION:}

ZUH: Made substantial contributions to the conception or design of the work; or the acquisition, analysis, or interpretation of data for the work; Drafted the work or revising it critically for important intellectual content; Approval final version to be published; Agreed to be account-able for all aspects of the work in ensuring that questions related to the accuracy or integrity of any part of the work are appropriately investigated and resolved. DK, SH, FZ, AMG, MH, JP: Made substantial contributions to the conception or design of the work; or the acquisition, analysis, or interpretation of data for the work; Approved final version to be published; Agreement to be accountable for all aspects of the work in ensuring that questions related to the accuracy or integrity of any part of the work are appropriately investigated and resolved. AH, YMY: Drafted the work or revised it critically for important intellectual content; Approved final version to be published; Agreed to be accountable for all aspects of the work in ensuring that questions related to the accuracy 
or integrity of any part of the work are appropriately investigated and resolved.

\section{REFERENCES}

1. Atiq MA. Epidemiology of non-communicable diseases in Pakistan: Are we on the right track? Pak J Med Dentistry 2018; 6:52-6.

2. Yusuf S, Hawken S, Ounpuu S, Dans T, Avezum A, Lanas F, et al. Effect of potentially modifiable risk factors associated with myocardial infarction in 52 countries (the INTERHEART study): case-control study. Lancet 2004; 364:537-52.

3. World Health Organization. Country cooperating strategy at glance. [Online].; May 2013 [cited 201508 13. Available from: http://www.who.int/countries/pak/en/].

4. UNDP, Human Development Report. [Online] 2013 [cited 2015 08 14. Available from: http://hdr.undp.org/en/reports/global/ hdr2013/download/]

5. Fletcher GF, Balady GJ, Amsterdam EA, Chaitman B, Eckel R, Fleg $\mathrm{J}$, et al. Exercise standards for testing and training: $\mathrm{A}$ statement for healthcare professionals from the American Heart Association. Circulation 2001; 104:1694-740.

6. Stone JA, Cyr C, Friesen M, Kennedy-Symonds H, Stene R, Smilovitch M. Canadian guidelines for cardiac rehabilitation and atherosclerotic heart disease prevention. Can J Cardiol 2001; 17 Suppl B:3B-30B.

7. Globe AJ, Worcester MUC. Best practice guidelines for cardiac rehabilitation \& secondary prevention. Victoria: Heart Research Center. [Online] 1999 [cited 201508 15. Available from: http:// rubens.its.unimelb.edu.au/heart/].

8. Jones DA, West PR. Psychological rehabilitation after myocardial infarction: Multicenter randomized control trial. BMJ 1996; 313:1517-21.

9. Rees K, Bennett P, West R, Smith GD, Ebrahim S. Psychological interventions for coronary heart disease. Cochrane Database Syst Rev 2004; 2:CD002902.

10. Haq ZU, Hisam A. Australian New Zealand Clinical Trials. Cited on 1st January 2018. Available at: https://www.anzctr.org.au/ Trial/Registration/TrialReview.aspx?id=372829\&isReview=true

11. Peixoto TC, Begot I, Bolzan DW, Machado L, Reis MS, Papa V, et al. Early exercise-based rehabilitation improves healthrelated quality of life and functional capacity after acute myocardial infarction: A randomized controlled trial. Can $\mathrm{J}$ Cardiol 2015; 31:308-13.

12. Roudijk B, Donders R, Stalmeier P. Cultural values: Can they explain self-reported health? Qual Life Res 2017; 26:1531-9.

13. Kashyap GC, Singh SK. Reliability and validity of general health questionnaire (GHQ-12) for male tannery workers: A study carried out in Kanpur, India. BMC Psychiatry 2017; 17:102.

14. Friedrich O, Sipötz J, Benzer W, Kunschitz E, Höfer S. The dimensional structure of the Macnew health-related quality of life questionnaire: A Mokken scale analysis. J Psychosom Res 2015; 79:43-8.

15. Cole TJ, Bellizzi MC, Flegal KM, Dietz WH. Establishing a standard definition for child overweight and obesity worldwide: International survey. BMJ 2000; 320:1240-3.

16. Bergman E, Malm D, Karlsson JE, Bertero C. Longitudinal study of patients after myocardial infarction: Sense of coherence, quality of life, and symptoms. Heart Lung 2009; 38:129-40.

17. Soto M, Failde I, Márquez S, Benítez E, Ramos I, Barba A, et al. Physical and mental component summaries score on the SF-36 in coronary patients. Qual Life Res 2005; 14:759-68.

18. Izawa K1, Hirano Y, Yamada S, Oka K, Omiya K, lijima S. Improvement in physiological outcomes and health-related quality of life following cardiac rehabilitation in patients with acute myocardial infarction. Circ J 2004; 68:315-20.

19. Grace SL, Abbey SE, Shnek ZM, Irvine J, Franche RL, Stewart DE. Cardiac rehabilitation II: Referral and participation. Gen Hosp Psychiatry 2002; 24:127-34.

20. Samartzis L, Dimopoulos S, Tziongourou M, Nanas S. Effect of psychosocial interventions on quality of life in patients with chronic heart failure: A meta-analysis of randomized controlled trials. J Card Fail 2013; 19:125-34

21. Chan DS, Chau JP, Chang AM. Acute coronary syndromes: Cardiac rehabilitation programmes and quality of life. J Adv Nurs 2005; 49:591-9.

22. Bettencourt N, Dias C, Mateus P, Sampaio F, Santos L, Adão L, et al. Impact of cardiac rehabilitation on quality of life and depression after acute coronary syndrome. Rev Port Cardio 2005; 24:687-96.

23. Taylor RS, Dalal H, Jolly K, Moxham T, Zawada A. Homebased versus centre-based cardiac rehabilitation. Cochrane Database Syst Rev 2010; 1:CD007130. 